\title{
Probe Cocktail to Assess Transporter Function in Sandwich-Cultured Human Hepatocytes
}

\author{
Cen Guo ${ }^{1}$, Kenneth R. Brouwer ${ }^{2}$, Paul W. Stewart ${ }^{3}$, Caroline Moseley², Kim L.R. Brouwer ${ }^{1}$ \\ 1. Division of Pharmacotherapy and Experimental Therapeutics, UNC Eshelman School of Pharmacy, University of North \\ Carolina at Chapel Hill, Chapel Hill, NC. ${ }^{2}$ BioIVT, ADME-TOX Division, Durham, NC. ${ }^{3}$ Department of Biostatistics, UNC \\ Gillings School of Public Health, University of North Carolina at Chapel Hill, Chapel Hill, NC.
}

Received, August 27, 2019; Revised, September 6, 2019; Accepted, October 28, 2019; Published, November 3, 2019.

\begin{abstract}
PURPOSE: Probe substrates are used routinely to assess transporter function in vitro. Administration of multiple probe substrates together as a "cocktail" in sandwich-cultured human hepatocytes $(\mathrm{SCHH})$ could increase the throughput of transporter function assessment in a physiologically-relevant in vitro system. This study was designed to compare transporter function between cocktail and single agent administration in SCHH. METHODS: Rosuvastatin, digoxin, and metformin were selected as probe substrates of hepatic transporters OATP1B1, OATP1B3, BCRP, P-gp, and OCT1. Total accumulation (Cells+Bile) and biliary excretion index (BEI) values derived from administration of the cocktail were compared to values obtained after administration of single agents in the absence and presence of a model inhibitor, erythromycin estolate. RESULTS: For rosuvastatin and metformin accumulation, the ratio of means [ $90 \%$ confidence interval (CI)] for cocktail to single agent administration was $100 \%$ [94\%, 106\%] and 90\% [82\%, 99\%], respectively. Therefore, the cocktail and single-agent mode of administration were deemed equivalent per standard equivalence criterion of $80-120 \%$ for rosuvastatin and metformin accumulation, but not for digoxin accumulation $(77 \%[62 \%, 92 \%])$. The ratio of means $[90 \% \mathrm{CI}]$ for rosuvastatin BEI values between the two administration modes $(105 \%$ [97\%, 114\%]) also was deemed equivalent. The ratio for digoxin BEI values between the two administration modes was $99 \%$ $[78 \%, 120 \%]$. In the presence of erythromycin estolate, the two administration modes were deemed equivalent for evaluation of rosuvastatin, digoxin, and metformin accumulation; the ratio of means [ $90 \% \mathrm{CI}]$ was $104 \%$ [ $94 \%$, $115 \%], 94 \%$ [82\%, 105\%], and 100\% [88\%, 111\%], respectively. However, rosuvastatin and digoxin BEI values were low and quite variable in the presence of the inhibitor, so the BEI results were inconclusive. CONCLUSIONS: These data suggest that rosuvastatin and metformin can be administered as a cocktail to evaluate the function of OATP1B1, OATP1B3, BCRP, and OCT1 in SCHH, and that digoxin may not be an ideal component of such a cocktail.
\end{abstract}

\section{INTRODUCTION}

Drug transporters have important implications in drug safety and efficacy(1). To evaluate the function of individual transporters, in vitro assays expressing single transporters are commonly used. The use of several probes administered together as a "cocktail" is a well-established, efficient approach to evaluate the activity of human drug-metabolizing cytochrome P450 enzymes(2). Such an approach is based on the simultaneous administration of probe substrates with minimal mutual interference at low concentrations. However, the application of transporter probe cocktails has been limited until recently(3) (4). Ebner et al. proposed a transporter cocktail containing the probe substrates digoxin, furosemide, metformin, and rosuvastatin(5). Data from transfected systems indicated a low likelihood of interactions between the probes, which is crucial to the utility of probe cocktails(5). However, in the follow-up clinical studies, the pharmacokinetics of rosuvastatin was altered by other components in this cocktail(6). When the clinical doses of metformin and furosemide were reduced, no mutual interaction was observed(7).

The poor correlation between the drug-drug interaction potential in vitro and in vivo could be attributed to the selected in vitro systems. Transfected systems typically lack the full complement of transporters and, therefore, the

Corresponding author: Kim L.R. Brouwer, UNC Eshelman School of Pharmacy, University of North Carolina at Chapel Hill, CB \#7569, Chapel Hill, NC 27599-7569, E-mail address: kbrouwer@unc.edu 
interaction between multiple transporters and probes in vivo may not be correctly predicted. A physiologically relevant in vitro system expressing multiple transporters would enable the simultaneous evaluation of different transporters when used in combination with a probe cocktail. Therefore, this system would increase the efficiency in assessing transporter function. Sandwich-cultured human hepatocytes (SCHH) are well-suited for the in vitro characterization of a transporter probe cocktail because they exhibit functional expression of multiple transporters responsible for the uptake and efflux of probe substrates by hepatocytes(8).

In this study, $\mathrm{SCHH}$ were used to evaluate a transporter probe cocktail containing rosuvastatin, digoxin, and metformin, which are substrates for multiple hepatic transporters of interest to drug development scientists and regulatory agencies including organic anion transporting polypeptide (OATP) 1B1, OATP1B3, breast cancer resistance protein (BCRP), P-glycoprotein (P-gp), and organic cation transporter 1(OCT1)(9-13). To investigate whether the cocktail administration could be used instead of individual administration of each probe, the two modes of administration were compared in terms of the disposition of each probe. Secondarily, because the performance of the cocktail in comparison to individual probes may be affected by the presence of an inhibitor, the comparison between the two administration modes also was performed in the presence of erythromycin estolate (EE), a model inhibitor for P-gp, OATP1B1, OATP1B3, and BCRP $(14,15)$ (Transporter Certified Datasheet published online by Qualyst Transporter Solutions).

\section{MATERIALS AND METHODS}

Please see Supporting Information for a detailed description of the Materials.

\section{Selection of Cocktail Probes}

Probe substrates for the cocktail were selected based on the following criteria: high metabolic stability, substrates for hepatic transporters of interest, minimal overlap of transporters, and clinical relevance. Based on these criteria, rosuvastatin (substrate of OATP1B1, OATP1B3, OATP2B1, MRP2, MRP4, BCRP)(9-11, 16), digoxin (substrate of P-gp)(13), and metformin (substrate of OCT1, MATE1)(12, 17) were selected. The dosing concentrations of the probes were: digoxin $0.5 \mu \mathrm{M}$, rosuvastatin $1 \mu \mathrm{M}$, and metformin $2 \mu \mathrm{M}$. These concentrations were chosen to minimize interference with other probes based on the inhibitory potential of each probe substrate against the transporters of other probes, and medium and cellular concentrations of each probe. In addition, the sensitivity of the bioanalytical methods was considered. The inhibitory potency and Michaelis-Menten constant of each probe towards its respective transporters are summarized in Table 1 in Supporting Information.

\section{Sandwich Cultured Human Hepatocytes (SCHH) and Disposition of Probe Substrates}

Cryopreserved human hepatocytes from three donors (HC3-26, FEA, IVL) were cultured in a sandwich configuration as previously described(18). Donors included two Caucasian females and one Asian female (age range: 43-57 years; body mass index range: 20-39). The disposition of each probe substrate in $\mathrm{SCHH}$ was evaluated when administered as a single agent or as a cocktail. B-CLEAR ${ }^{\circledR}$ technology (Qualyst Transporter Solutions, BioIVT, Durham, NC) was used to estimate the biliary excretion index (BEI) of each probe substrate, which was calculated using the equation below $(8,19)$.

BEI $(\%)=\frac{\text { Amount }_{\text {Cells }+ \text { Bile }}-\text { Amou }}{\text { Cells }} \times 100$

Additional details regarding hepatocyte culture and assessment of probe disposition in $\mathrm{SCHH}$ are described in Supporting Information. Each experiment was performed in triplicate wells, unless otherwise specified, and repeated using hepatocytes from three donors. As a secondary investigation, the same experiment was performed in the presence of $\mathrm{EE}(100 \mu \mathrm{M})$, a model inhibitor for P-gp, OATP1B1, OATP1B3, and BCRP $(14,15)$ (personal communication [Transporter Certified Datasheet published online by Qualyst Transporter Solutions using XenoTech, LLC human hepatocytes]). The measurement of probe substrates is described in Supporting Information.

\section{STATISTICAL ANALYSIS}

The primary outcome variables for analysis were the amount of probe in Cells+Bile and the BEI value of the probe, which were analyzed in linear scale using a repeated-measures analysis of variance model. The primary variables were analyzed as a function of 
administration mode (single, cocktail), with hepatocyte donor as the random effect. The model was used to obtain point- and interval-estimates of the two means and the ratio of the two means. Single and cocktail modes were considered equivalent if the $90 \%$ confidence interval (CI) for the ratio of the two means (mean for cocktail mode/mean for singleagent mode) fell entirely within $80-120 \%(20)$. The accumulation data were analyzed separately for each of the six scenarios: probe substrate (rosuvastatin, digoxin, metformin) $\times$ EE inhibitor (absent, present). The BEI data were analyzed separately for each of the four scenarios: probe substrate (digoxin, metformin) $\times \mathrm{EE}$ inhibitor (absent, present). For the three instances in which the measured amount of probe in Cells+Bile was less than that in Cells alone, a value of zero was imputed for the BEI value. The statistical methods are consistent with recommendations described previously $(21,22)$. See Supporting Information for additional details.

\section{RESULTS}

Primary study results: comparison of cocktail and single agent in the absence of EE (Table 1)

The amount of rosuvastatin in Cells+Bile (total accumulation) between cocktail and single-agent mode was similar across all three hepatocyte donors (Figure 1A). The estimate of the ratio of the two means (i.e., cocktail mean divided by single-agent mean) was $100 \%$, and the $90 \%$ CI fell entirely within the well-established bioequivalence range of 80 $120 \%$. The estimates of rosuvastatin BEI values for cocktail and single-agent mode were similar, and the $90 \% \mathrm{CI}$ for the ratio of the means fell within the accepted bioequivalence range.

Table 1. Accumulation (total amount in Cells+Bile) and Biliary Excretion Index (BEI) for probe substrates after single agent or cocktail administration (in the absence of erythromycin estolate).

\begin{tabular}{|c|c|c|c|}
\hline \multicolumn{4}{|c|}{ Accumulation in Cells+Bile (pmol/mg protein) } \\
\hline Probe substrate & $\begin{array}{c}\text { Mean for } \\
\text { Single Mode }\end{array}$ & $\begin{array}{c}\text { Mean for } \\
\text { Cocktail Mode }\end{array}$ & $\begin{array}{c}\text { Ratio }^{a} \\
\text { of Means (\%) }\end{array}$ \\
\hline Rosuvastatin & $\begin{array}{c}49.51 \\
{[3.00,96.02]^{b}}\end{array}$ & $\begin{array}{c}49.56 \\
{[3.05,96.07]^{b}}\end{array}$ & $\begin{array}{c}100 \\
{[94,106]^{c}}\end{array}$ \\
\hline Digoxin & $\begin{array}{c}8.88 \\
{[-0.54,18.30]}\end{array}$ & $\begin{array}{c}6.82 \\
{[-2.60,16.24]}\end{array}$ & $\begin{array}{c}77 \\
{[62,92]}\end{array}$ \\
\hline Metformin & $\begin{array}{c}7.00 \\
{[-1.13,15.14]}\end{array}$ & $\begin{array}{c}6.33 \\
{[-1.80,14.47]}\end{array}$ & $\begin{array}{c}90 \\
{[82,99]}\end{array}$ \\
\hline \multicolumn{4}{|c|}{ BEI (\%) } \\
\hline Probe substrate & $\begin{array}{c}\text { Mean for } \\
\text { Single Mode }\end{array}$ & $\begin{array}{c}\text { Mean for } \\
\text { Cocktail Mode }\end{array}$ & $\begin{array}{c}\text { Ratio }^{a} \\
\text { of Means (\%) }\end{array}$ \\
\hline Rosuvastatin & $\begin{array}{c}52.00 \\
{[24.9,79.1]^{b}}\end{array}$ & $\begin{array}{c}54.67 \\
{[27.6,81.7]^{b}}\end{array}$ & $\begin{array}{c}105 \\
{[97,114]^{c}}\end{array}$ \\
\hline Digoxin & $\begin{array}{c}38.00 \\
{[27.5,48.5]}\end{array}$ & $\begin{array}{c}37.67 \\
{[27.2,48.2]}\end{array}$ & $\begin{array}{c}99 \\
{[78,120]}\end{array}$ \\
\hline Metformin & $\mathrm{NA}^{d}$ & NA & NA \\
\hline \multicolumn{4}{|c|}{$\begin{array}{l}\left.{ }^{a} \text { Ratio = (mean for cocktail) / (mean for single agent }\right) \times 100 \% \\
b 95 \% \text { confidence interval for the mean to convey level of precision } \\
{ }^{c} 90 \% \text { confidence interval for the ratio to evaluate equivalence } \\
{ }^{d} \text { Not Available: biliary excretion of metformin is negligible. }\end{array}$} \\
\hline
\end{tabular}


A

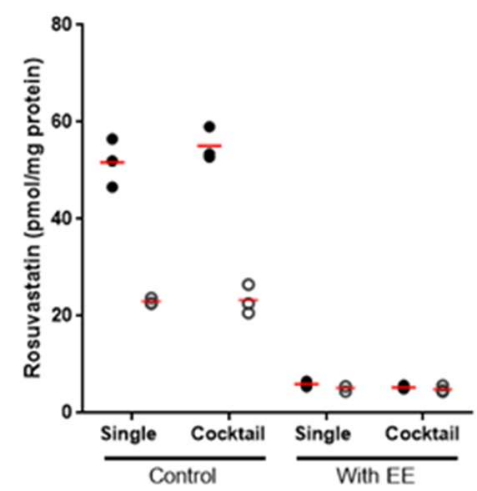

\begin{tabular}{|l|l|l|l|l|}
\hline BEI (\%) & 55 & 58 & 13 & 6.5 \\
\hline
\end{tabular}

B

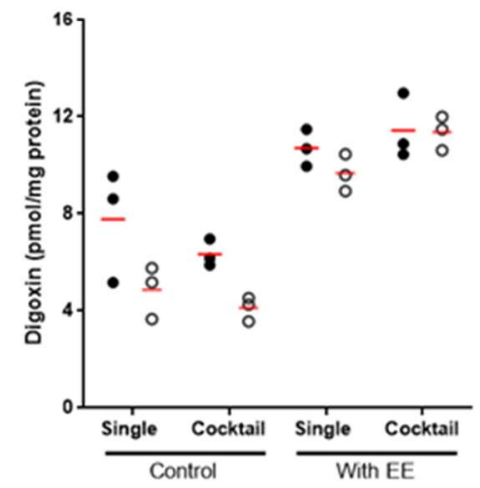

\begin{tabular}{|c|c|c|c|c|}
\hline BEI (\%) & 37 & 35 & 10 & 0.64 \\
\hline
\end{tabular}

C

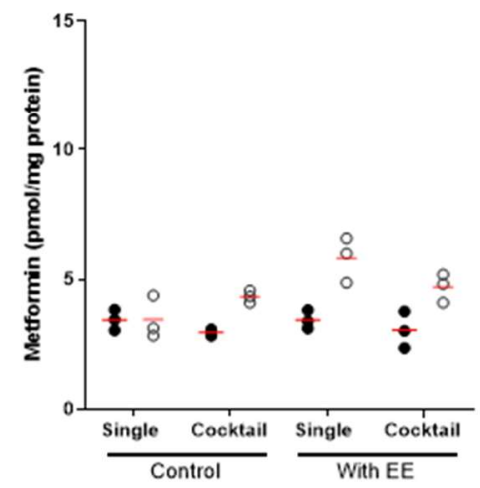

FEA

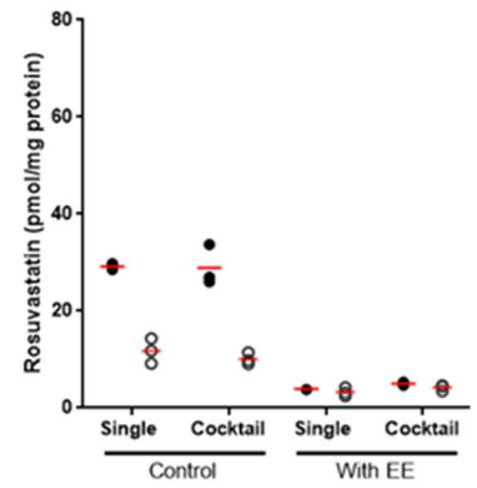

\begin{tabular}{|l|l|l|l|l|}
\hline BEI (\%) & 60 & 65 & 17 & 17 \\
\hline
\end{tabular}

FEA

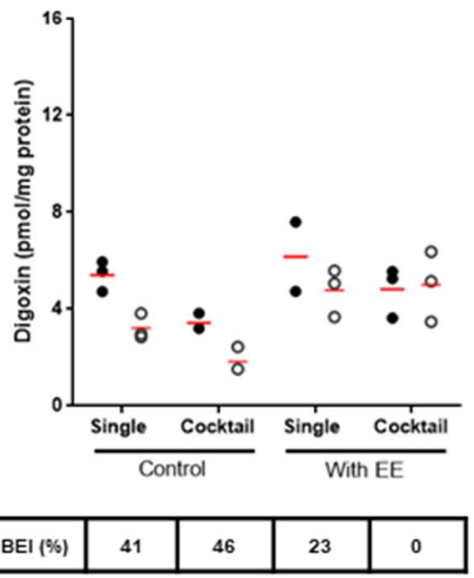

FEA

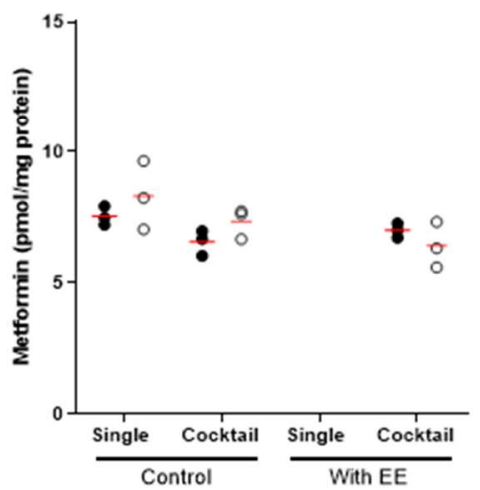

IVL

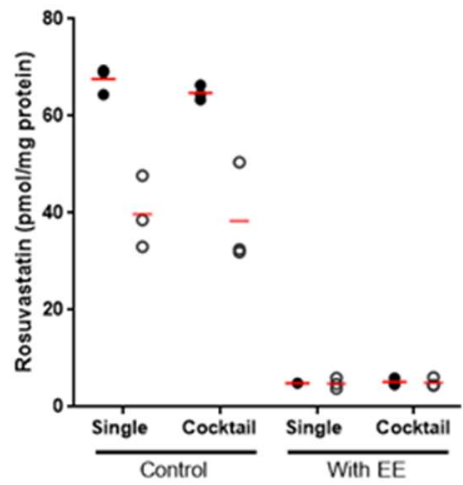

\begin{tabular}{|l|l|l|l|l|}
\hline BEI (\%) & 41 & 41 & 1.7 & 2.0 \\
\hline
\end{tabular}

IVL

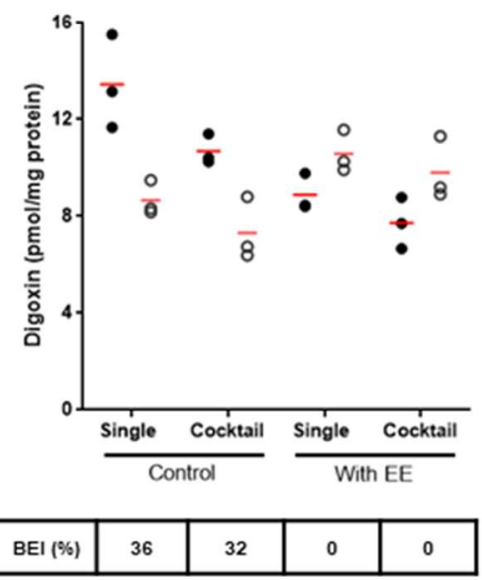

IVL

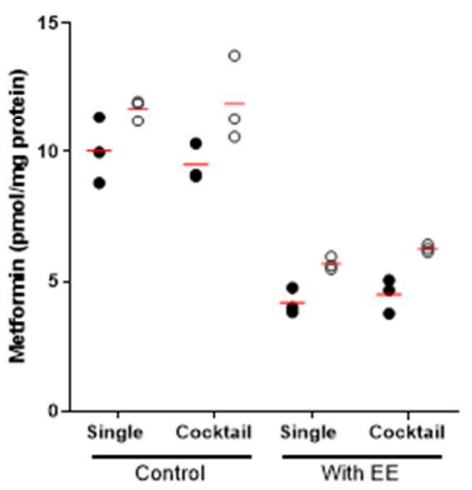

Figure 1. Disposition of (A) rosuvastatin, (B) digoxin, and (C) metformin in sandwich-cultured human hepatocytes administered as a single agent or as a cocktail in the absence (control) or presence of erythromycin estolate (EE). Studies were repeated in three individual hepatocyte donors: HC3-26, FEA, IVL. Solid and open circles represent the amount of probe substrate in Cells+Bile and Cells, respectively, in each well. Data for metformin uptake administered individually in the presence of EE are not available for hepatocyte donor FEA. Red lines represent arithmetic means of the triplicate measurements. Biliary excretion index (BEI) values of rosuvastatin and digoxin for each group are shown under the figures. Statistical analyses of the data are summarized in Tables 1 and 2. 
Digoxin accumulation was slightly lower when administered as a cocktail than as a single agent (Figure 1B). The $90 \%$ CI for the ratio of the two means did not fall entirely within the $80-120 \%$ interval, and therefore, the evaluation of bioequivalence was inconclusive. The estimates of the means of digoxin BEI for the cocktail and singleagent mode were in close agreement; the $90 \%$ CI for the ratio of the two means was very close to being within the $80-120 \%$ interval.

Metformin accumulation was comparable between cocktail and single-agent mode (Figure 1C). The estimate of the $90 \%$ CI of the ratio of the two means satisfied the equivalence criterion. As expected, the biliary excretion of metformin was negligible, therefore, BEI values were not calculated
Secondary study results: comparison of cocktail and single agent in the presence of EE (Table 2)

Rosuvastatin total accumulation values (Figure 1A) were similar for the two modes when EE was present, and the estimate and $90 \% \mathrm{CI}$ of the ratio of the two means satisfied the equivalence criterion. The means of rosuvastatin BEI values for the cocktail and single-agent mode were 8.50 and $10.57 \%$, respectively, in the presence of EE; BEI values were classified as low (less than $\sim 15 \%$ ) and variable, as evidenced by the wide $95 \% \mathrm{CI}$. The $90 \%$ CI of the ratio of the two means did not satisfy the equivalence criterion, and therefore, the results were inconclusive.

Table 2. Accumulation (total amount in Cells+Bile) and Biliary Excretion Index (BEI) for probe substrates after single agent or cocktail administration (in the presence of erythromycin estolate).

\begin{tabular}{|c|c|c|c|}
\hline \multicolumn{4}{|c|}{ Accumulation in Cells + Bile ( $\mathrm{pmol} / \mathrm{mg}$ protein) } \\
\hline Probe substrate & $\begin{array}{c}\text { Mean for } \\
\text { Single Mode }\end{array}$ & $\begin{array}{c}\text { Mean for } \\
\text { Cocktail Mode }\end{array}$ & $\begin{array}{c}\text { Ratio }^{a} \\
\text { of Means (\%) }\end{array}$ \\
\hline Rosuvastatin & $\begin{array}{c}4.93 \\
{[3.75,6.10]^{b}}\end{array}$ & $\begin{array}{c}5.15 \\
{[3.97,6.32]^{b}}\end{array}$ & $\begin{array}{c}104 \\
{[94,115]^{c}}\end{array}$ \\
\hline Digoxin & $\begin{array}{c}8.54 \\
{[1.82,15.26]}\end{array}$ & $\begin{array}{c}7.99 \\
{[1.20,14.78]}\end{array}$ & $\begin{array}{c}94 \\
{[82,105]}\end{array}$ \\
\hline Metformin & $\begin{array}{c}4.86 \\
{[0.21,9.52]}\end{array}$ & $\begin{array}{c}4.84 \\
{[0.038,9.65]}\end{array}$ & $\begin{array}{c}100 \\
{[88,111]}\end{array}$ \\
\hline \multicolumn{4}{|c|}{ BEI $(\%)$} \\
\hline Probe substrate & $\begin{array}{l}\text { Mean for } \\
\text { Single Mode }\end{array}$ & $\begin{array}{c}\text { Mean for } \\
\text { Cocktail Mode }\end{array}$ & $\begin{array}{c}\text { Ratio }^{a} \\
\text { of Means (\%) }\end{array}$ \\
\hline Rosuvastatin & $\begin{array}{c}10.57 \\
{[-6.89,28.0]^{b}}\end{array}$ & $\begin{array}{c}8.50 \\
{[-8.95,25.95]^{b}}\end{array}$ & $\begin{array}{c}80 \\
{[20,141]^{c}}\end{array}$ \\
\hline Digoxin & $\begin{array}{c}11.00 \\
{[-2.08,24.08]}\end{array}$ & $\begin{array}{c}0.21 \\
{[-12.87,13.29]}\end{array}$ & $\begin{array}{c}2 \\
{[-123,127]}\end{array}$ \\
\hline Metformin & $\mathrm{NA}^{d}$ & NA & NA \\
\hline \multicolumn{4}{|c|}{$\begin{array}{l}{ }^{a} \text { Ratio }=(\text { mean for cocktail) } / \text { (mean for single agent }) \times 100 \% \\
{ }^{b} 95 \% \text { confidence interval for the mean to convey level of precision } \\
{ }^{c} 90 \% \text { confidence interval for the ratio to evaluate equivalence } \\
{ }^{d} \text { Not Available: biliary excretion of metformin is negligible. }\end{array}$} \\
\hline
\end{tabular}


Digoxin total accumulation values (Figure 1B) yielded similar estimates for the means in the cocktail and single-agent mode when EE was present. The estimate and $90 \% \mathrm{CI}$ for the ratio of means satisfied the equivalence criterion. Digoxin BEI values in the presence of EE were low and variable (Figure 1B). The estimate of the mean BEI value for the cocktail mode was much lower than for the single-agent mode in the presence of EE $(0.64 \%$ vs $10 \%, 0 \%$ vs $23 \%$, and $0 \%$ vs $0 \%$ ). Although the point estimate for the ratio of the means was $2 \%$, the $90 \%$ CI was outside of the equivalence range, indicating inconclusive results.

Metformin total accumulation values (Figure 1C) yielded practically identical estimates for cocktail and single-agent mode in the presence of EE. The point estimate and $90 \%$ CI for the ratio of means satisfied the equivalence criterion. Accumulation data for the single-agent mode from donor FEA were missing due to a technical error. BEI values were not calculated because the biliary excretion of metformin was negligible, as expected.

\section{DISCUSSION}

This study examined the disposition of each probe substrate following cocktail administration compared to single-agent administration. The primary outcome measures were accumulation in Cells+Bile and BEI, which were analyzed to assess the function of uptake and biliary transporters. When $90 \% \mathrm{CI}$ of the ratio of means fell entirely within the interval $80-120 \%$, the two groups were deemed equivalent. This is the standard criterion used in clinical bioequivalence studies (80-120\%) for linearscale data(20). The conclusions of this study were based on the accumulation results in the absence and presence of EE, and BEI results in the absence of EE. The BEI values of rosuvastatin and digoxin in the presence of $\mathrm{EE}$ were low and quite variable, and therefore, the results were inconclusive.

In the case of rosuvastatin, the two modes of administration were deemed equivalent, which was expected. These results indicated that the transporters involved in the hepatic uptake and biliary excretion of rosuvastatin did not appear to be affected by digoxin and metformin used in this cocktail. Therefore, our study suggests that the function of OATP1B1, OATP1B3, BCRP, and MRP4 can be assessed in SCHH using this cocktail approach without interference by the other probes administered in this cocktail. This observation was in line with the clinical study by Stopfer et al., where $50 \mathrm{mg}$ metformin $\left(\mathrm{C}_{\max } \sim 1 \mu \mathrm{M}\right)$ did not change rosuvastatin pharmacokinetics(7).

In the absence of EE, digoxin accumulation was lower for the cocktail mode than for the single-agent mode, although the dosing concentrations of rosuvastatin and metformin used in this study were not expected to affect the disposition of digoxin mediated by P-gp. Digoxin is used widely as a P-gp probe substrate, however, other transporters are involved in the hepatic disposition of digoxin, including OATP1B3(23), sodium-dependent pathways(24), and organic solute transporter alpha/beta $(\operatorname{OST} \alpha / \beta)(25,26)$. The activity of these transporters may have varied across the hepatocyte donors, or transporter function may have been inhibited or stimulated by other components of the cocktail. Therefore, digoxin may not be an optimal probe to include in this cocktail for SCHH studies.

In control hepatocytes, metformin accumulation in Cells+Bile between cocktail and single-agent mode met the equivalence criteria, which was expected. These results indicated that the active uptake of metformin mediated by OCT 1 could be studied using this cocktail without interference by other probes. Although metformin is also a substrate for canalicular transporters MATE1 and MATE2K(17), the expression levels of these transporters are low in $\mathrm{SCHH}(27)$, which may explain the minimal biliary excretion of metformin in $\mathrm{SCHH}$.

A secondary objective of this study was to evaluate the performance of the probe cocktail in the presence of a model inhibitor. In the presence of EE, the accumulation of rosuvastatin, digoxin and metformin in Cells+Bile all met the equivalence criteria. In this study, EE decreased the uptake of rosuvastatin, which is consistent with inhibition of OATP1B(15). EE also decreased rosuvastatin BEI, consistent with previous findings with rosuvastatin and pravastatin in SCHH (Transporter Certified Datasheet published online by Qualyst Transporter Solutions). EE decreased digoxin BEI values, which is consistent with reports that EE inhibited P-gp in vitro (14). There was no obvious impact of $\mathrm{EE}$ on metformin accumulation in hepatocyte lot HC3-26 and FEA, while metformin uptake was decreased in hepatocyte lot IVL. This inter-donor difference could be attributed to the variable expression level of OCT1 in human hepatocytes(28), which is representative of the total expression in human liver $(28,29)$. The effect of EE on OCT1 has not been 
reported and could not be established based on the results of the present study. Future studies are warranted to assess the effect of inhibitors and inducers once a suitable transporter probe cocktail is identified.

Inter-donor variability was observed in terms of the effect of drug administration mode and EE treatment. Transporter activity may vary among donors due to individual differences (e.g., expression of functional protein on the basolateral and canalicular membranes, driving forces). Recent experience in screening over 200 lots of cryopreserved human hepatocytes from multiple vendors indicated that only $\sim 60 \%$ of the lots examined demonstrated transporter function equivalent to fresh primary human hepatocytes evaluated under the same conditions (BioIVT internal data). This low equivalence rate may explain some variability in experiments using cryopreserved human hepatocytes, as observed in the present data.

Methodology that increases the efficiency of characterizing transporter function could improve the utility of in vitro models to predict metabolic induction, hepatotoxicity, and transporter interactions. Compared to transfected cell lines or membrane vesicles, the use of SCHH and probe cocktails to assess transporter function and identify potential interactions among probes may be more predictive of in vivo data. Probe substrates can interact with uptake and/or efflux transporters of other probes in the cocktail. Therefore, simultaneous expression of multiple uptake and efflux transporters would be needed, but is lacking in single-transfected systems. The evaluation of transporter cocktails in SCHH allows a more holistic view of the potential interactions among probes and provides support for clinical dose selection.

In summary, the hepatic accumulation of the probe substrates rosuvastatin and metformin in SCHH were equivalent between cocktail and singleagent mode in the absence and presence of EE. The $\mathrm{BEI}$ of rosuvastatin also was equivalent between the two modes in the absence of EE. Therefore, rosuvastatin and metformin could serve as base components for the development of transporter probe cocktails containing additional probe substrates, while digoxin may not be an ideal component of such a cocktail. This SCHH transporter probe cocktail assay is a rapid, straightforward, and cost-effective approach to assess transporter function in a physiologicallyrelevant in vitro system. This approach can be used to identify other transporter probe cocktail combinations that may be useful for $\mathrm{SCHH}$ and in vivo studies.

\section{ABBREVIATIONS}

$\mathrm{BCRP}$, breast cancer resistance protein; BEI, biliary excretion index; $\mathrm{CI}$, confidence interval; $\mathrm{EE}$, erythromycin estolate; OATP, organic anion transporting polypeptide; OST $\alpha / \beta$, organic solute transporter alpha/beta; OCT, organic cation transporter; P-gp, P-glycoprotein; SCHH, sandwichcultured human hepatocytes

\section{ACKNOWLEDGEMENTS}

This work was supported by the National Institute of General Medical Sciences of the National Institutes of Health (NIH) under Award Number R35 GM122576 (K.L.R.B), BioIVT, the Brewington Research Award and the Royster Society of Fellows (C.G.).

\section{CONFLICT OF INTEREST}

Dr. Kim L.R. Brouwer is co-inventor of the sandwich-cultured hepatocyte technology for quantification of biliary excretion (B-CLEAR ${ }^{\circledR}$ ) and related technologies, which have been licensed exclusively to Qualyst Transporter Solutions, now BioIVT. Dr. Kenneth R. Brouwer and Caroline Moseley are employees of BioIVT, which has exclusively licensed the B-CLEAR ${ }^{\circledR}$ technology.

\section{REFERENCES}

1. Giacomini, K.M. and S.M. Huang, Transporters in drug development and clinical pharmacology. Clinical pharmacology and therapeutics, 2013; 94(1):3-9.

2. Dierks, E.A., et al., A method for the simultaneous evaluation of the activities of seven major human drug-metabolizing cytochrome P450s using an in vitro cocktail of probe substrates and fast gradient liquid chromatography tandem mass spectrometry. Drug metabolism and disposition: the biological fate of chemicals, 2001; 29(1):23-9.

3. Prueksaritanont, T., et al., Validation of a microdose probe drug cocktail for clinical drug interaction assessments for drug transporters and CYP3A. Clinical pharmacology and therapeutics, 2017; 101(4):519-530. 
4. Kosa, R.E., et al., Simultaneous assessment of transporter-mediated drug-drug interactions using a probe drug cocktail in cynomolgus monkey. Drug metabolism and disposition: the biological fate of chemicals, 2018; 46(8):1179-1189.

5. Ebner, T., N. Ishiguro, and M.E. Taub, The use of transporter probe drug cocktails for the assessment of transporter-based drug-drug interactions in a clinical setting-proposal of a four component transporter cocktail. Journal of pharmaceutical sciences, 2015; 104(9):3220-8.

6. Stopfer, P., et al., Pharmacokinetic evaluation of a drug transporter cocktail consisting of digoxin, furosemide, metformin, and rosuvastatin. Clinical pharmacology and therapeutics, 2016; 100(3):259-67.

7. Stopfer, P., et al., Effects of metformin and furosemide on rosuvastatin pharmacokinetics in healthy volunteers: implications for their use as probe drugs in a transporter cocktail. European journal of drug metabolism and pharmacokinetics, 2018; 43(1):69-80.

8. Yang, K., et al., Sandwich-cultured hepatocytes as a tool to study drug disposition and drug-induced liver injury. Journal of pharmaceutical sciences, 2016; 105(2):443-59.

9. Ho, R.H., et al., Drug and bile acid transporters in rosuvastatin hepatic uptake: function, expression, and pharmacogenetics. Gastroenterology, 2006; 130(6): 1793-806.

10. Deng, J.W., et al., Effect of silymarin supplement on the pharmacokinetics of rosuvastatin. Pharmaceutical research, 2008; 25(8):1807-14.

11. Pfeifer, N.D., K. Yang, and K.L.R. Brouwer, Hepatic basolateral efflux contributes significantly to rosuvastatin disposition I: characterization of basolateral versus biliary clearance using a novel protocol in sandwich-cultured hepatocytes. The Journal of pharmacology and experimental therapeutics, 2013; 347(3):727-36.

12. Choi, M.K., et al., Effects of tetraalkylammonium compounds with different affinities for organic cation transporters on the pharmacokinetics of metformin. Biopharmaceutics \& drug disposition, 2007; 28(9):501-10.

13. Lee, C.A., et al., ITC commentary on the prediction of digoxin clinical drug-drug interactions from in vitro transporter assays. Clinical pharmacology and therapeutics, 2014; 96(3):298-301.

14. Eberl, S., et al., Role of p-glycoprotein inhibition for drug interactions: evidence from in vitro and pharmacoepidemiological studies. Clinical pharmacokinetics, 2007; 46(12):1039-49.

15. De Bruyn, T., et al., Structure-based identification of OATP1B1/3 inhibitors. Molecular pharmacology, 2013; 83(6):1257-67.

16. Kanda, K., et al., Total hepatocellular disposition profiling of rosuvastatin and pitavastatin in sandwich- cultured human hepatocytes. Drug metabolism and pharmacokinetics, 2018; 33(3):164-172.

17. Tanihara, Y., et al., Substrate specificity of MATE1 and MATE2-K, human multidrug and toxin extrusions/H(+)-organic cation antiporters. Biochemical pharmacology, 2007; 74(2):359-71.

18. Guo, C., et al., Farnesoid $\mathrm{X}$ receptor agonists obeticholic acid and chenodeoxycholic acid increase bile acid efflux in sandwich-cultured human hepatocytes: functional evidence and mechanisms. The Journal of pharmacology and experimental therapeutics, 2018; 365(2):413-421.

19. Liu, X., et al., Correlation of biliary excretion in sandwich-cultured rat hepatocytes and in vivo in rats. Drug metabolism and disposition: the biological fate of chemicals, 1999; 27(6):637-44.

20. Rasheed, R. and A.A. Siddiqui, Use of geometric mean in bioequivalence trials. Int J Stats Med Res 2015:6.

21. Wasserstein, R.L. and N.A. Lazar, The ASA's statement on p-values: context, process, and purpose. The American Statistician, 2016; 70(2):129-133.

22. Wasserstein, R.L., A.L. Schirm, and N.A. Lazar, Moving to a world beyond " $\mathrm{p}<0.05$ ". The American Statistician, 2019; 73(sup1):1-19.

23. Kullak-Ublick, G.A., et al., Organic aniontransporting polypeptide B (OATP-B) and its functional comparison with three other OATPs of human liver. Gastroenterology, 2001; 120(2):525-33.

24. Taub, M.E., et al., Digoxin is not a substrate for organic anion-transporting polypeptide transporters OATP1A2, OATP1B1, OATP1B3, and OATP2B1 but is a substrate for a sodium-dependent transporter expressed in HEK293 cells. Drug metabolism and disposition: the biological fate of chemicals, 2011; 39(11):2093-102.

25. Seward, D.J., et al., Functional complementation between a novel mammalian polygenic transport complex and an evolutionarily ancient organic solute transporter, OSTalpha-OSTbeta. The Journal of biological chemistry, 2003; 278(30):27473-82.

26. Malinen, M.M., et al., Organic solute transporter OSTalpha/beta is overexpressed in nonalcoholic steatohepatitis and modulated by drugs associated with liver injury. Am J Physiol Gastrointest Liver Physiol, 2018; 314(5):G597-g609.

27. Schaefer, M., et al., Quantitative expression of hepatobiliary transporters and functional uptake of substrates in hepatic two-dimensional sandwich cultures: a comparative evaluation of upcyte and primary human hepatocytes. Drug metabolism and disposition: the biological fate of chemicals, 2018; 46(2):166-177.

28. Schaefer, O., et al., Absolute quantification and differential expression of drug transporters, cytochrome P450 enzymes, and UDPglucuronosyltransferases in cultured primary human 
hepatocytes. Drug metabolism and disposition: the biological fate of chemicals, 2012; 40(1):93-103.

29. Kumar, V., et al., A comparison of total and plasma membrane abundance of transporters in suspended, plated, sandwich-cultured human hepatocytes versus human liver tissue using quantitative targeted proteomics and cell surface biotinylation. Drug metabolism and disposition: the biological fate of chemicals, 2019; 47(4):350-357. 